\title{
MÉTODOS DE IMAGEM EMPREGADOS NO DIAGNÓSTICO DAS ALTERAÇÕES DA ARTICULAÇÃO TEMPOROMANDIBULAR: UMA REVISÃO DE LITERATURA
}

\author{
IMAGING METHODS IN TEMPOROMANDIBULAR ARTICULATION \\ CHANGES: A LITERATURE REVIEW
}

\section{Ana Aparecida de Almeida Pereira', Jamille Rios Moura' ${ }^{2}$, Neyldes Moreira da Silva ${ }^{3}$}

\author{
Graduada no curso de Tecnologia em Radiologia pela Faculdade Estácio Feira de Santana. \\ Feira de Santana, Bahia, Brasil. anaaparecida.radiologia@hotmail.com \\ ${ }^{2}$ Autora para correspondência. Mestre em Saúde Coletiva. Docente na Faculdade Estácio Feira de Santana. \\ Feira de Santana, Bahia, Brasil. jamillerios19@yahoo.com.br \\ ${ }^{3}$ Graduada em Tecnologia em Radiologia. Docente na Faculdade Estácio Feira de Santana. \\ Feira de Santana, Bahia, Brasil. neyldes.com@hotmail.com
}

RESUMO | Introdução: Diante da sua complexidade anatômica, a articulação temporomandibular (ATM) é uma das regiões corpóreas que oferece maior dificuldade à obtenção de imagens convencionais. Nesse contexto, se faz necessária a utilização de exames imaginológicos que possuam uma maior eficácia para contribuir na obtenção do diagnóstico de alterações dessa articulação. Objetivo: o presente artigo tem por objetivo estudar a eficácia dos exames imaginológicos empregados no diagnóstico das alterações e/ou lesões da articulação temporomandibular (ATM). Material e métodos: trata-se de uma revisão da literatura, na qual foram selecionados dez artigos. As bases de dados PubMed e Scielo foram consultadas. Utilizaram-se os seguintes descritores: articulação temporomandibular, tomografia, imagem por ressonância magnética, diagnóstico por imagem e tomografia computadorizada de feixe cônico. Resultados: A radiografia panorâmica foi considerada um método de imagem contra indicado para o diagnóstico de alterações na ATM, devido às distorções das imagens. A ressonância magnética foi reconhecida como padrão ouro para avaliar alterações nos tecidos moles da ATM. Para avaliar alterações ósseas da ATM, a tomografia computadorizada cone beam foi considerada ideal. Conclusão: cada método de imagem possui indicação específica para o diagnóstico de alterações na ATM. Contudo, ao conciliar as imagens de ressonância magnética e tomografia computadorizada cone beam, ambas proporcionam um diagnóstico complementar de todas as estruturas que compõem essa articulação.

Palavras-chave: Articulação temporomandibular; Tomografia; Imagem por ressonância magnética; Diagnóstico por imagem; tomografia computadorizada de feixe cônico.

\begin{abstract}
Introduction: Due to its anatomical complexity, the temporomandibular joint (TMJ) is one of the corporeal regions that offers greater difficulty in obtaining conventional images. In this context, it is necessary to use imaging exams that have a greater effectiveness in order to contribute to the diagnosis of alterations of this joint. Objective: this article aims to study the efficacy of imaging tests used to diagnose temporomandibular joint (TMJ) changes and / or injuries. Material and methods: it is a review of the literature, in which ten articles were selected. The PubMed and Scielo databases were consulted. The following descriptors were used: temporomandibular joint, tomography, magnetic resonance imaging, diagnostic imaging and cone-beam computed tomography. Results: Panoramic radiography was considered as an imaging method, not indicated for the diagnosis of TMJ changes due to distortions of the images. Magnetic resonance imaging was recognized as a gold standard for assessing soft tissue changes in TMJ. To evaluate bone changes of the TMJ, cone beam computed tomography was considered ideal. Conclusion: Each imaging method has a specific indication for the diagnosis of TMJ changes. However, in reconciling magnetic resonance imaging and cone beam computed tomography, both provide a complementary diagnosis of all the structures that make up this joint.
\end{abstract}

Keywords: temporomandibular joint, tomography, magnetic resonance imaging, diagnostic imaging and cone-beam computed tomography. 


\section{INTRODUÇÃO}

A Articulação Temporomandibular (ATM) é uma articulação sinovial constituída de tecidos moles e estruturas ósseas, responsável por conectar a mandíbula ao osso temporal e de promover a realização de todos os movimentos da cavidade oral $^{1,2}$. As estruturas ósseas que compõe a ATM são cabeça do côndilo, fossa glenóide e eminência articular do osso temporal. Dentre os tecidos moles, estão os músculos da mastigação, os ligamentos articulares, o disco articular e a cápsula articular ${ }^{3}$.

A ATM tem sido frequentemente acometida por alguns fatores que interferem diretamente em suas funções, o que tem contribuído assim para 0 surgimento das disfunções temporomandibulares (DTM) ${ }^{4}$. Doenças, alterações e/ou lesões podem interferir no funcionamento correto dessa articulação, tais como, artrite de origem infecciosa e traumática, neoplasias e anquiloses. Porém, o deslocamento da mandíbula, fraturas condilares e anquilose, estão entre os principais fatores que levam os pacientes a realização dos exames de imagem, no intuito de diagnosticar e solucionar tais problemas'.

Diante da sua complexidade anatômica, a ATM é uma das articulações do corpo humano que apresentam maior dificuldade à aquisição de imagens convencionais, por ser uma articulação de tamanho pequeno e parcialmente encoberta pelas densas estruturas ósseas do crânio, acarretando assim sobreposições em suas imagens. Com isso, se faz necessário a utilização de exames imaginológicos que possuem uma maior eficácia para contribuir na obtenção do diagnóstico, através da visualização mais detalhada e das informações mais precisas que esses exames trazem dessa articulação ${ }^{5}$.

Dentre os exames solicitados para diagnosticar possíveis alterações e/ou lesões que acometem a ATM, a Ressonância Magnética (RM), a Tomografia Computadorizada (TC) e a Tomografia computadorizada Cone Beam (TCCB), são considerados como métodos de imagem ideal quando comparados aos exames convencionais. Os exames imaginológicos convencionais, a saber, a radiografia panorâmica, não são eficazes no diagnóstico de possíveis alterações da ATM pelo fato dos mesmos apresentarem distorções nas imagens e dificuldade de interpretação $(6,7)$.
A RM tem sido muito utilizada para o diagnóstico de possíveis alterações e/ou lesões da ATM por ser considerado um exame de imagem que proporciona especificidade, sensibilidade e uma maior precisão na reprodução de estruturas anatômicas articulares ${ }^{6}$. A TC é classificada como um excelente exame de imagem para $\circ$ estudo das estruturas ósseas da ATM, pois possibilita uma visualização tridimensional de todos os detalhes ósseos sem que haja sobreposições dos mesmos, proporcionando o tamanho e o formato real das estruturas anatômicas, das variações morfológicas e das condições patológicas dessa articulação ${ }^{3}$. A TCCB traz como uma de suas vantagens o menor tempo de exposição à radiação no momento da realização do exame, como também uma execução mais rápida do mesmo, o que se configura em um grande benefício para o paciente, além de auxiliar no diagnóstico de possíveis alterações e/ou lesões da ATM através da visualização minuciosa das estruturas dessa articulação, permitindo também aquisições de imagens de excelente precisão após a sua reconstruçãob.

Nesse âmbito, destaca-se que o presente estudo tem como relevância auxiliar os profissionais da saúde na escolha do exame de imagem ideal para diagnosticar possíveis alterações da ATM de acordo com os sintomas do paciente, tendo como proposta, obter um diagnóstico preciso das alterações encontradas nessa articulação, evitando assim, exposições desnecessárias do paciente a radiação.

Diante do exposto, o presente artigo tem por objetivo estudar a eficácia dos exames imaginológicos empregados no diagnóstico das alterações e/ou lesões da ATM.

\section{METODOLOGIA}

Trata-se de um estudo bibliográfico de revisão literária, com o objetivo de fundamentar teoricamente os diferentes métodos de imagem empregados no diagnóstico das alterações da articulação temporomandibular.

Para o desenvolvimento deste estudo foram consultadas duas bases de dados: Public Medline 
(PubMed) e Scientific Electronic Library Online (Scielo). Estabeleceram-se como critérios de inclusão artigos publicados nos idiomas português e inglês, cujos textos encontravam-se disponíveis na íntegra; estudos publicados no período entre janeiro de 2015 a setembro de 2016; artigos baseados em exames imaginológicos de Ressonância Magnética (RM), Radiografia Panorâmica (RP), Tomografia Computadorizada (TC) e Tomografia Computadorizada de Cone Beam (TCCB); e estudos que avaliam possíveis alterações e/ou lesões na Articulação Temporomandibular (ATM).

Como critérios de exclusão definiram-se: estudos não descritos nos idiomas referidos, não disponíveis na íntegra, publicados há mais de dois anos e que não apresentassem relação com o tema abordado.

Foi iniciada a busca nas referidas bases de dados, utilizando descritores de pesquisa cadastrados no Descritores em Ciências da Saúde (Decs). As palavras-chave utilizadas foram: articulação temporomandibular, tomografia, imagem por ressonância magnética, diagnóstico por imagem e tomografia computadorizada de feixe cônico. Tais descritores foram empregados nos idiomas inglês e português, quando aplicados na base de dados PubMed e Scielo, respectivamente. Foram utilizados os operadores booleanos AND e OR para combinar as palavras-chave mencionadas acima. As combinações dos descritores foram aplicadas em ambas as bases de dados, a fim de obter um maior número de artigos relacionados ao tema de interesse.
Na base de dados PubMed foram aplicadas três strings de busca. Na primeira foram utilizados os seguintes descritores: temporomandibular joint, temporomandibular joint disorders e diagnostic imaging. Um total de quatro mil quatrocentos e dois artigos foram encontrados, dos quais trinta e oito obedeciam aos critérios de inclusão. Após a leitura na íntegra destes artigos, quatro foram selecionados para compor a revisão. A segunda string envolveu os descritores temporomandibular joint, temporomandibular joint disorders, tomography e cone-beam computed tomography. Encontrou-se um total de onze mil quinhentos e dezessete artigos, dos quais duzentos e setenta obedeciam aos critérios de inclusão, sendo selecionados destes dois para compor o estudo. Na elaboração da terceira string foram empregados os seguintes descritores: temporomandibular joint, temporomandibular joint disorders e magnetic resonance imaging. Foi identificado um total de um mil e oitocentos artigos, dos quais dezesseis obedeciam aos critérios de inclusão. Destes, três foram selecionados, após a leitura na íntegra, para compor a revisão.

Na base de dados Scielo foi elaborada uma string de busca, na qual os seguintes descritores foram utilizados: articulação temporomandibular e diagnóstico por imagem. Encontrou-se um total de oito artigos, dos quais seis obedeciam aos critérios de inclusão. Após a leitura destes na íntegra, apenas um foi selecionado para compor o estudo.

Quadro 1. Panorama indicativo da combinação dos descritores

\begin{tabular}{|c|c|c|c|c|}
\hline $\begin{array}{c}\text { Base } \\
\text { de } \\
\text { dados }\end{array}$ & Descritores & $\begin{array}{c}\mathbf{N}^{\circ} \text { total de } \\
\text { artigos } \\
\text { encontrados }\end{array}$ & $\begin{array}{c}\mathbf{N}^{\circ} \text { de artigos } \\
\text { levantados }\end{array}$ & $\begin{array}{c}\mathbf{N}^{\circ} \text { de artigos } \\
\text { selecionados } \\
\text { para a revisão }\end{array}$ \\
\hline \multirow{2}{*}{ PUBMED } & $\begin{array}{c}\text { Temporomandibular Joint OR } \\
\text { Temporomandibular Joint } \\
\text { Disorders AND Diagnostic } \\
\text { Imaging }\end{array}$ & $\begin{array}{c}\text { Temporomandibular Joint OR } \\
\text { Temporomandibular Joint } \\
\text { Disorders AND Tomography } \\
\text { OR Cone-Beam Computed } \\
\text { Tomography }\end{array}$ & 11.517 & 38 \\
\cline { 2 - 5 } & $\begin{array}{c}\text { Temporomandibular Joint OR } \\
\text { Temporomandibular Joint } \\
\text { Disorders AND Magnetic } \\
\text { Resonance Imaging }\end{array}$ & 1.800 & 270 & 2 \\
\hline \multirow{2}{*}{ SCIELO } & $\begin{array}{c}\text { Articulação } \\
\text { temporomandibular AND } \\
\text { diagnóstico por imagem }\end{array}$ & 8 & 6 & 3 \\
\hline
\end{tabular}




\section{RESULTADOS E DISCUSSÃO}

Foram selecionados dez artigos, através das buscas eletrônicas, para a elaboração desse estudo. Estes foram comparados quanto o autor, revista, ano, objetivo geral, resultados e conclusão, conforme a apresentação no quadro sinóptico (Quadro 2).

Pode-se observar que em relação à origem, os estudos foram procedentes dos seguintes países: Brasil, Canadá, Índia, República de Latvia e Suiça. No que se refere ao idioma, todos os dez artigos mencionados foram escritos na língua inglesa.

Ao realizar o levantamento dos artigos pode-se observar que quatro estudos eram transversais, três revisões de literatura, um ensaio clínico, um estudo de caso e uma revisão sistemática.

Em relação aos resultados dos trabalhos incluídos na revisão, considera-se que a indicação correta de um exame de imagem para a avaliação de alterações na Articulação Temporomandibular (ATM) seja baseada a partir da necessidade do paciente, levando em consideração seus sinais e sintomas, avaliados por meio de exame clínico inicial $^{8}$. Da mesma forma, alguns autores relatam que apesar de ser considerado um método de suma importância, o exame clínico não tem sido suficiente para estabelecer um diagnóstico incontestável das alterações que acometem a ATM, devido as suas limitações. Porém, juntamente com ○ auxílio dos exames imaginológicos, ambos estabelecem um método eficaz de diagnóstico, proporcionando dados complementares da região da ATM, sejam de alterações morfológicas e/ou condições patológicas que irão contribuir para a obtenção de um diagnóstico mais preciso dessas alterações ${ }^{5}$.

No que se refere ao uso da Radiografia Panorâmica (RP) para o diagnóstico de alterações na ATM, um estudo relata que a RP é utilizada tanto para avaliar quanto para diagnosticar inicialmente a ATM, porém quando se refere às alterações morfológicas dessa articulação, a RP, pelo fato de apresentar distorções nas imagens, é contra indicada para tal análise, pois poderá sugerir um falso diagnóstico (9). Em contrapartida, deformações ósseas avançadas no côndilo, como fraturas, alterações de crescimento, anquiloses, tumores, e grandes alterações morfológicas do tubérculo articular, podem ser identificadas através da $\mathrm{RP}^{8}$. As limitações deste exame estão atreladas às inúmeras estruturas ósseas presentes no crânio, as quais impedem obter uma boa visualização das estruturas dessa articulação, devido à sobreposição de estruturas anatômicas ${ }^{6,7}$.

Um estudo realizado com crianças portadoras de artrite idiopática juvenil, também descreve que a RP é ineficaz para $\circ$ diagnóstico de pequenas lesões ósseas presentes no côndilo da ATM ${ }^{10}$. Além disso, outros autores verificaram em seu estudo que, no que se refere ao diagnóstico de alterações na ATM de pacientes com outro tipo de artrite, a RP não possui clareza em suas imagens devido as sobreposição e distorções que a mesma apresenta ${ }^{11}$. Contudo, mesmo apresentando vantagens tais como - baixo custo, baixa irradiação e fácil execução, a RP somente possibilita a visualização de lesões tardiamente, ou seja, quando estas atingem um estágio avançado de degradação óssea ${ }^{12}$.

Outros exames de imagem têm sido utilizados na odontologia para o diagnóstico de alterações da ATM. Um estudo transversal realizado na Paraíba afirmou que a Ressonância Magnética (RM) é uma modalidade de imagem considerada padrão ouro para avaliar os discos da ATM, e que traz como vantagens um método não invasivo e $\circ$ não uso da radiação ionizante ${ }^{13}$. Além disso, outro estudo aponta que, apesar da ausência de radiação ionizante na realização do exame de RM, o mesmo disponibiliza, para a formação de suas imagens, de um forte campo magnético acoplado em um sistema de emissão e recepção de ondas e um computador, trazendo imagens de alta fidelidade e definição ${ }^{14}$.

Semelhantemente, outro estudo realizado no Canadá relata a efetividade que a RM apresenta no que se refere à avaliação da posição e morfologia da ATM e do disco articular ${ }^{15}$. Ademais, a RM é tida como um exame padrão ouro para a representação dos tecidos moles e determinação da posição do disco articular da ATM, permitindo informações sobre a posição, função e forma desse disco e condições dos tecidos musculares e ligamentos, além da avaliação da severidade de várias patologias ${ }^{6}$.

Alguns autores destacaram o exame de RM como uma modalidade de imagem ideal na avaliação precisa da determinação da posição do disco articular, como também sua morfologia ${ }^{16}$. Da mesma forma, Kumar 
et al. (2015) enfatizam a capacidade da RM de permitir a visualização do disco e de proporcionar informações sobre a posição morfológica, a intensidade do sinal e a estrutura desse disco nos movimentos de abertura e fechamento da boca, em pacientes assintomáticos e sintomáticos com deslocamento do disco articular ${ }^{17}$. Semelhantemente, outro estudo realizado na Suíça ressalta a eficácia da RM de demonstrar a presença de inflamações ativas e alterações envolvendo a ATM em pacientes com artrite idiopática juvenil ${ }^{18}$. Além disso, para diagnosticar alterações intracapsulares da ATM, no que se refere ao deslocamento mandibular, podese considerar a RM como um excelente exame de imagem no diagnóstico de tal patologia ${ }^{19}$.

Uma revisão sistemática relata que mesmo com todo - avanço da capacidade de diagnóstico da RM, a mesma apresenta baixa qualidade em suas imagens, quando se refere ao estudo dos componentes ósseos da ATM $^{20}$. Da mesma forma, Gonçalves, Dotta e Serra (2011) afirmam que ainda é possível encontrar limitações desse exame no diagnóstico das alterações da ATM, pois a mesmo não disponibiliza de clareza de detalhes e nem de precisão dos componentes ósseos dessa articulação, sendo assim necessário o auxílio de outros exames de imagens para o estudo aprofundado desses componentes e para a obtenção de um diagnóstico mais completo de toda a estrutura articular da ATM ${ }^{14}$.

Outros métodos de imagem utilizados para - diagnóstico de alterações da ATM são a Tomografia Computadorizada (TC) e a Tomografia Computadorizada Cone Beam (TCCB). Segundo Ferreira et al. (2016) são métodos de imagem extremamente superiores aos exames convencionais, como a RP, permitindo um diagnóstico preciso das estruturas ósseas da ATM por meio de imagens observadas nos planos sagital, coronal e axial, as quais permitem passarem por manipulações $e$ reconstruções tridimensionais, através de softwares específicos $^{8}$. A TCCB quando comparado a TC, traz como uma de suas vantagens o menor tempo de exposição à radiação no momento da realização do exame, como também a execução mais rápida do mesmo, o que se configura como um grande benefício para o paciente 6 .

De acordo com Al-Shwaikh et al. (2016), além da TCCB possibilitar a obtenção de imagens em três dimensões e a realização das mesmas em um menor tempo e com uma menor dose de radiação, quando comparado a TC, permite também a partir dessas imagens, uma avaliação precisa da morfologia e das alterações da ATM ${ }^{10}$. Da mesma forma, um estudo de caso realizado no Brasil descreve que a TCCB é um método preciso para avaliar os componentes ósseos e as condições morfológicas das estruturas ósseas da ATM?.

Alguns autores ressaltam que a TCCB possui a capacidade de demonstrar achatamento da cabeça do côndilo, caracterizando-se como uma patologia que mais acomete as estruturas ósseas da ATM em pacientes crianças com artrite idiopática juvenil $^{20}$. Em contrapartida, a TCCB limita-se apenas a avaliação dos componentes ósseos, e ao diagnóstico de alterações presentes nas estruturas ósseas da ATM, ou seja, o mesmo não é indicado para avaliar tecidos moles dessa articulaçãa ${ }^{21}$. Dessa forma, quando for necessária a avaliação por imagem dos tecidos moles, nos casos de lesões, o exame de escolha deve ser inicialmente a RM, assim como, para diagnosticar possíveis alterações e/ou lesões da ATM, a TCCB será indicada somente quando essas alterações estiverem comprometendo os componentes ósseos dessa articulação ${ }^{22}$.

Entretanto, Al-Saleh et al. (2016) afirmam que para o diagnóstico de possíveis alterações na ATM, ambos os métodos de imagem, a RM e TCCB, se complementam entre si para obter um diagnóstico completo das alterações dessa articulação ${ }^{20}$. Além disso, alguns autores destacam que, mesmo apresentando limitações, a RM e a TCCB representam, quando associados, métodos de imagem importantes para diagnosticar alterações tanto nos componentes ósseos quanto nos tecidos moles da ATM $^{8}$. Do mesmo modo, ao conciliar as imagens da RM com as da TCCB, ambas proporcionam um diagnóstico complementar de todas as estruturas que compõem a $\mathrm{ATM}^{15}$.

Portanto, diante dos diferentes tipos de exames imaginológicos apresentados, é fundamental que os profissionais que atuam na área de diagnóstico e tratamento das desordens da ATM busquem capacitação, através de cursos de aperfeiçoamento, atualização ou, até mesmo, especialização, a fim de se qualificar para a correta indicação de exames de imagens na avaliação de possíveis alterações da 
ATM, associando os seus conhecimentos aos sinais e sintomas apresentados pelo paciente.

Na constituição desse estudo houve limitações, as quais se restringiram aos métodos de pesquisa. Essa deficiência foi decorrente de alguns fatores tais como, a maioria dos estudos serem de idioma inglês, e poucos desenvolvidos no Brasil, o que indica a falta de interesse dos brasileiros, inclusive dos profissionais de odontologia, quanto à área de imaginologia e radiologia odontológica.

Quadro 2. Quadro Sinóptico dos estudos selecionados para a revisão.

\begin{tabular}{|c|c|c|c|c|c|}
\hline AUTORES & REVISTA/ANO & $\begin{array}{l}\text { TIPO DE } \\
\text { ESTUDO }\end{array}$ & OBJETIVO GERAL & RESULTADOS & CONCLUSÃO \\
\hline $\begin{array}{l}\text { Ferreira LA et } \\
\text { al. }\end{array}$ & $\begin{array}{l}\text { Braz J } \\
\text { Otorhinolaryngol / } \\
2016\end{array}$ & $\begin{array}{l}\text { Revisão de } \\
\text { literatura }\end{array}$ & $\begin{array}{l}\text { Apresentar e discutir os } \\
\text { principais exames de imagem no } \\
\text { diagnóstico de distúrbios da } \\
\text { articulação temporomandibular, } \\
\text { como também seus critérios de } \\
\text { indicação, vantagens e } \\
\text { desvantagens. }\end{array}$ & $\begin{array}{l}\text { A tomografia } \\
\text { computadorizada e a } \\
\text { ressonância magnética foram } \\
\text { apontadas como padrão ouro } \\
\text { para avaliação dos tecidos } \\
\text { duros e tecidos moles da } \\
\text { articulação } \\
\text { temporomandibular. }\end{array}$ & $\begin{array}{l}\text { Destaca-se a importância da } \\
\text { indicação adequada dos exames } \\
\text { a serem solicitados, para que se } \\
\text { obtenha um diagnóstico preciso } \\
\text { da articulação } \\
\text { temporomandibular. }\end{array}$ \\
\hline $\begin{array}{l}\text { Al-Shwaikh et } \\
\text { al. }\end{array}$ & $\begin{array}{l}\text { Stomatologija, } \\
\text { Baltic Dental and } \\
\text { Maxillofacial } \\
\text { Journal/ } 2016\end{array}$ & $\begin{array}{l}\text { Estudo } \\
\text { transversal }\end{array}$ & $\begin{array}{l}\text { Avaliar e comparar as } \\
\text { características radiológicas dos } \\
\text { componentes ósseos da } \\
\text { articulação temporomandibular } \\
\text { em pacientes com e sem artrite } \\
\text { idiopática juvenil por meio da } \\
\text { Tomografia Computadorizada } \\
\text { Cone Beam. }\end{array}$ & $\begin{array}{l}\text { Quando comparados os grupos } \\
\text { com artrite juvenil idiopática e } \\
\text { o controle, observou-se que as } \\
\text { características radiológicas das } \\
\text { estruturas ósseas ocorreram } \\
\text { assimetricamente entre os } \\
\text { lados direito e esquerdo da } \\
\text { articulação } \\
\text { temporomandibular. }\end{array}$ & $\begin{array}{l}\text { As imagens de Tomografia } \\
\text { Computadorizada Cone Beam } \\
\text { mostraram uma maior } \\
\text { predominância de características } \\
\text { de destruição óssea da } \\
\text { articulação temporomandibular } \\
\text { nos grupos com artrite juvenil } \\
\text { idiopática e uma menor } \\
\text { frequência no grupo controle. }\end{array}$ \\
\hline Al-Saleh ef al. & $\begin{array}{l}\text { Journal of } \\
\text { Otolaryngology/ } \\
2016\end{array}$ & $\begin{array}{l}\text { Revisão } \\
\text { sistemática }\end{array}$ & $\begin{array}{l}\text { Analisar através da literatura a } \\
\text { importância das imagens de } \\
\text { ressonância magnética, } \\
\text { tomografia computadorizada e } \\
\text { Tomografia Computadorizada } \\
\text { Cone Beam para a avaliação } \\
\text { da anatomia da articulação } \\
\text { temporomandibular. }\end{array}$ & $\begin{array}{l}\text { Dentre os artigos, somente três } \\
\text { atenderam os critérios de } \\
\text { inclusão. Onde dois destes } \\
\text { descrevia sobre ressonância } \\
\text { magnética e um sobre } \\
\text { tomografia computadorizada. }\end{array}$ & $\begin{array}{l}\text { A quantidade de estudos sobre } \\
\text { ressonância magnética, } \\
\text { tomografia computadorizada e } \\
\text { Tomografia Computadorizada } \\
\text { Cone Beam, dificultaram a } \\
\text { conclusão quanto à precisão } \\
\text { desses exames na avaliação da } \\
\text { articulação temporomandibular. }\end{array}$ \\
\hline Sodhi, et al. & $\begin{array}{l}\text { Contemporary } \\
\text { Clinical Dentistry/ } \\
2016\end{array}$ & $\begin{array}{l}\text { Estudo de } \\
\text { caso }\end{array}$ & $\begin{array}{l}\text { Destacar a função das imagens } \\
\text { de Tomografia } \\
\text { Computadorizada Cone Beam } \\
\text { no diagnóstico das alterações } \\
\text { ósseas na fase inicial da artrite } \\
\text { reumatóide na articulação } \\
\text { temporomandibular. }\end{array}$ & $\begin{array}{l}\text { Devido às sobreposições das } \\
\text { estruturas ósseas da } \\
\text { articulação } \\
\text { temporomandibular, foi } \\
\text { realizada uma avaliação mais } \\
\text { detalhada dessa articulação } \\
\text { por meio da Tomografia } \\
\text { Computadorizada Cone Beam. }\end{array}$ & $\begin{array}{l}\text { A Tomografia Computadorizada } \\
\text { Cone Beam revelou com mais } \\
\text { precisão mudanças degenerativas } \\
\text { precoces na articulação } \\
\text { temporomandibular. Com isso, foi } \\
\text { tido como um exame eficaz no } \\
\text { diagnóstico desta doença } \\
\text { degenerativa. }\end{array}$ \\
\hline $\begin{array}{l}\text { Ladeira DBS, } \\
\text { Cruz AD, } \\
\text { Almeida SM }\end{array}$ & $\begin{array}{l}\text { Original Research } \\
\text { Imaginology/ } 2015\end{array}$ & $\begin{array}{l}\text { Estudo } \\
\text { transversal }\end{array}$ & $\begin{array}{l}\text { Avaliar a eficácia da } \\
\text { radiografia panorâmica digital, } \\
\text { comparando-a com as imagens } \\
\text { de Tomografia } \\
\text { Computadorizada Cone Beam } \\
\text { como padrão ouro para o } \\
\text { diagnóstico de transtornos } \\
\text { morfológicos da articulação } \\
\text { temporomandibular. }\end{array}$ & $\begin{array}{l}\text { Houve uma grande variedade } \\
\text { nos resultados das alterações } \\
\text { morfológicas da articulação } \\
\text { temporomandibular } \\
\text { diagnosticadas pela } \\
\text { radiografia panorâmica } \\
\text { digital. }\end{array}$ & $\begin{array}{l}\text { A radiografia panorâmica digital } \\
\text { não tem eficiência para o } \\
\text { diagnóstico das mudanças } \\
\text { morfológicas na articulação } \\
\text { temporomandibular. }\end{array}$ \\
\hline $\begin{array}{l}\text { Al- } \\
\text { Saleh et al. }\end{array}$ & $\begin{array}{l}\text { Dentomaxillofacial } \\
\text { Radiology/ } 2015\end{array}$ & $\begin{array}{l}\text { Revisão de } \\
\text { literatura }\end{array}$ & $\begin{array}{l}\text { Comparar a qualidade da } \\
\text { imagem dos exames de } \\
\text { ressonância magnética e } \\
\text { Tomografia Computadorizada } \\
\text { Cone Beam em relação à } \\
\text { anatomia e anormalidades } \\
\text { ósseas da articulação } \\
\text { temporomandibular. }\end{array}$ & $\begin{array}{l}\text { Nas imagens individuais de } \\
\text { ressonância magnética, foi } \\
\text { observada uma menor } \\
\text { frequência da posição do } \\
\text { disco, quando comparado com } \\
\text { as imagens de ressonância } \\
\text { magnética somada com as da } \\
\text { Tomografia Computadorizada } \\
\text { Cone Beam. }\end{array}$ & $\begin{array}{l}\text { Para identificar anormalidades } \\
\text { ósseas e a posição do disco e do } \\
\text { côndilo, as imagens de } \\
\text { ressonância magnética somadas } \\
\text { com as da Tomografia } \\
\text { Computadorizada Cone Beam } \\
\text { foram mais concretas que as } \\
\text { imagens de Tomografia } \\
\text { Computadorizada Cone Beam } \\
\text { individualmente. }\end{array}$ \\
\hline Keller et al. & $\begin{array}{l}\text { Pediatric } \\
\text { Rheumatology/ } \\
2015\end{array}$ & $\begin{array}{l}\text { Estudo } \\
\text { transversal }\end{array}$ & $\begin{array}{l}\text { Avaliar os achados clínicos } \\
\text { associados com a artrite precoce } \\
\text { na articulação } \\
\text { temporomandibular a partir da } \\
\text { ressonância magnética } \\
\text { contrastada. }\end{array}$ & $\begin{array}{l}\text { A associação da avaliação } \\
\text { clínica com a ressonância } \\
\text { magnética obtiveram um maior } \\
\text { percentual de sinais } \\
\text { envolvendo a articulação } \\
\text { temporomandibular. }\end{array}$ & $\begin{array}{l}\text { A avaliação clínica não permite } \\
\text { diagnosticar artrite precoce na } \\
\text { articulação temporomandibular } \\
\text { sem a presença da ressonância } \\
\text { magnética. }\end{array}$ \\
\hline
\end{tabular}


Quadro 2. Quadro Sinóptico dos estudos selecionados para a revisão. (continuação)

\begin{tabular}{|c|c|c|c|c|c|}
\hline AUTORES & REVISTA/ANO & $\begin{array}{l}\text { TIPO DE } \\
\text { ESTUDO }\end{array}$ & OBJETIVO GERAL & RESULTADOS & CONCLUSÃO \\
\hline Kumar et al. & $\begin{array}{l}\text { The Open Dentistry } \\
\text { Journal/ } \\
2015\end{array}$ & Ensaio clínico & $\begin{array}{l}\text { Analisar a correlação do } \\
\text { deslocamento do disco com a } \\
\text { presença ou ausência de sinais e } \\
\text { sintomas clínicos de distúrbios da } \\
\text { articulação temporomandibular } \\
\text { em pacientes sintomáticos e } \\
\text { assintomáticos, através das } \\
\text { imagens de ressonância } \\
\text { magnética. }\end{array}$ & $\begin{array}{l}\text { Tanto no grupo de pacientes } \\
\text { sintomáticos quanto no de } \\
\text { assintomáticos, foi encontrado, } \\
\text { por meio das imagens de } \\
\text { ressonância magnética, a } \\
\text { correlação do deslocamento } \\
\text { do disco com a presença ou } \\
\text { ausência de sinais e sintomas } \\
\text { clínicos de distúrbios da } \\
\text { articulação } \\
\text { temporomandibular. }\end{array}$ & $\begin{array}{l}\text { As imagens de ressonância } \\
\text { magnética demonstram com } \\
\text { precisão a correlação do } \\
\text { deslocamento do disco com a } \\
\text { presença ou ausência de sinais e } \\
\text { sintomas clínicos de distúrbios da } \\
\text { articulação temporomandibular. }\end{array}$ \\
\hline Farias et al. & $\begin{array}{l}\text { Dentomaxillofac } \\
\text { Radiol/ } 2015\end{array}$ & $\begin{array}{l}\text { Estudo } \\
\text { transversal }\end{array}$ & $\begin{array}{l}\text { Avaliar por meio da ressonância } \\
\text { magnética as estruturas do disco } \\
\text { e do côndilo da articulação } \\
\text { temporomandibular, bem como } \\
\text { a sua ligação com o } \\
\text { deslocamento do disco. }\end{array}$ & $\begin{array}{l}\text { Observou-se uma associação } \\
\text { entre a morfologia do disco e } \\
\text { o deslocamento do mesmo. }\end{array}$ & $\begin{array}{l}\text { A partir do exame de ressonância } \\
\text { magnética, os resultados deste } \\
\text { estudo demonstram que a } \\
\text { morfologia do disco da } \\
\text { articulação temporomandibular } \\
\text { está relacionada com o } \\
\text { deslocamento de disco. }\end{array}$ \\
\hline Larheim et al. & $\begin{array}{l}\text { DentomaxillofacialR } \\
\text { adiology/ } 2015\end{array}$ & $\begin{array}{l}\text { Revisão de } \\
\text { literatura }\end{array}$ & $\begin{array}{l}\text { Comparar o exame de } \\
\text { Tomografia Computadorizada } \\
\text { Cone Beam com outras } \\
\text { modalidades de exames para a } \\
\text { avaliação da articulação } \\
\text { temporomandibular em } \\
\text { diferentes categorias de } \\
\text { pacientes. }\end{array}$ & $\begin{array}{l}\text { A Tomografia } \\
\text { Computadorizada Cone Beam } \\
\text { é um exame de imagem } \\
\text { superior a exames } \\
\text { radiográficos convencionais. } \\
\text { Porém, limita-se ao diagnóstico } \\
\text { apenas dos componentes } \\
\text { ósseos, sendo a ressonância } \\
\text { magnética o exame indicado } \\
\text { para a avaliação de } \\
\text { anormalidades do tecido mole. }\end{array}$ & $\begin{array}{l}\text { Para a avaliação de desarranjos } \\
\text { dos tecidos moles e dos } \\
\text { componentes ósseos da } \\
\text { articulação temporomandibular, a } \\
\text { ressonância magnética e a } \\
\text { Tomografia Computadorizada } \\
\text { Cone Beam, respectivamente, são } \\
\text { métodos superiores às } \\
\text { radiografias convencionais. }\end{array}$ \\
\hline
\end{tabular}

\section{CONCLUSÃO}

A literatura consultada aponta que a radiografia panorâmica não deve ser aplicada para avaliar alterações morfológicas ou pequenas lesões ósseas presentes no côndilo mandibular. Trata-se de um método de diagnóstico em duas dimensões, o que pode gerar sobreposições, causadas pelas estruturas anatômicas da ATM e, consequentemente, distorções da imagem. A ressonância magnética é considerada padrão ouro para avaliar alterações presentes no disco articular, ligamentos e musculatura da ATM e demais alterações que acometem os tecidos moles dessa articulação. Nos casos de avaliação das estruturas ósseas da ATM, a tomografia computadorizada convencional ou cone beam são considerados exames de imagens ideais. Dessa forma, para um diagnóstico eficaz é necessário, em alguns casos, a combinação dos exames de ressonância magnética e tomografia computadorizada, pois ambos se complementam obtendo um diagnóstico preciso das alterações de todas as estruturas que compõem a ATM.

\section{CONFLITOS DE INTERESSES}

Nenhum conflito financeiro, legal ou político envolvendo terceiros (governo, empresas e fundações privadas, etc.) foi declarado para nenhum aspecto do trabalho submetido (incluindo mas não limitandose a subvenções e financiamentos, conselho consultivo, desenho de estudo, preparação de manuscrito, análise estatística, etc).

\section{REFERÊNCIAS}

1. Gutierrez LMO, Grossmann TK, Grossmann E. Deslocamento anterior da cabeça da mandíbula: diagnóstico e tratamento. Rev Dor. 2011 ; 1 2(1):64-70. doi: 10.1590/S1806$\underline{00132011000100014}$

2. Martins JS, Campos BM, Nahás-Scocate ACR, Fuziy A, de Freitas CF e Costa ALF. Avaliação do volume do disco articular da ATM por meio de imagens de ressonância magnética usando um software de análise de imagem. Rev Odontol Univ Cid. 2015;27(2):1 18-25.

3. Silveira OS, Silva FCS, de Almeida CEN, Tuji FM, Seraidarian PI, Manzi FR. Utilização da tomografia computadorizada para o diagnóstico da articulação temporomandibular. Rev CEFAC. 2014;16(6):2053-9. doi: $10.1590 / 1982-0216201418013$ 
4. Pachioni FSM, Aoyama AY, Pavia A, Pernas DL, Savian NU, Fregonesi CEPT et al. Disfunção temporomandibular: análise cefalométrica e fotogrametria. ConScientiae Saúde. 2013;1 2(2):177-84. doi: 10.5585/ConsSaude.v12n2.4139

5. Silveira OS, Silva FCS, de Almeida CEN, Tuji FM, Seraidarian Pl, Manzi FR. Utilização da tomografia computadorizada para o diagnóstico da articulação temporomandibular. Rev CEFAC. 2014; 16(6): 2053-9. doi: $10.1590 / 1982-0216201418013$

6. Machado E, Gresh RA, Cunali PA. Imaginologia da articulação temporomandibular durante o tratamento ortodôntico: uma revisão sistemática. Dental Press J Orthod. 2011 ; 16(3):54.e1-7. doi: 10.1590/S2176$\underline{94512011000300005}$

7. Rodrigues GHC, Rodrigues VA, de Barros SM, Ximenez MEL, de Souza DM. Correlação entre as Medidas Lineares em Radiografias Panorâmicas e Tomografias Computadorizadas Cone Beam Associadas ao Seio Maxilar. Pesq Bras Odontoped Clin Integr. 2013;13(3):245-9. doi: 10.4034/ PBOCl.2013.133.04

8. Ferreira LA, Grossmann E, Januzzi E, de Paula MVQ, Carvalho ACP. Diagnosis of temporomandibular joint disorders: indication of imaging exams. Braz J Otorhinolaryngol. 2016;82(3):341-52. doi: 10.1016/i. bjorl.2015.06.010

9. Ladeira DBS, da Cruz AD, de Almeida SM. Digital panoramic radiography for diagnosis of the temporomandibular joint: $\mathrm{CBCT}$ as the gold standard. Braz Oral Res [online]. $2015 ; 29(1): 1-7$. doi: 10.1590/18073107BOR-2015.vol29.0120

10. Al-Shwaikh H, Urtane I, Pirttiniemi P, Pesonen P, Krisjane Z, Jankovska I et al. Radiologic features of temporomandibular joint osseous structures in children with juvenile idiopathic arthritis. Cone beam computed tomography study. Stomatologija. 2016;18(2):51-60.

11. Sodhi A, Naik S, Pai A, Anuradha A. Rheumatoid arthritis affecting temporomandibular joint. Contemp Clin Dent. 2015;6(1):1 24-7. doi: 10.4103/0976-237X.149308

12. de Carvalho RT, Brag FSFF, Brito F, Junior JC, Figueredo CM, Sztajnbok FR. Alterações da articulação temporomandibular e suas repercussões orofaciais em pacientes portadores de artrite idiopática juvenil. Rev Bras Reumatol. 201 2;52(6):903-11. doi: 10.1590/S0482$\underline{50042012000600009}$

13. Farias JFG, Melo SLS, Bento PM, Oliveira LSAF, Campos PSF, de Melo DP. Correlation between temporomandibular joint morphology and disc displacement by MR.

Dentomaxillofac Radiol. 2015:44(7). doi: 10.1259/ dmfr.20150023
14. Gonçalves PE, Dotta EAV, Serra MC. Imageologia na odontologia e aspectos legais; RGO - Rev Gaúcha Odontol. $2011 ; 59: 89-95$.

15. Al-Saleh MA, Jaremko JL, Alsufyani N, Jibri Z, Lai $\mathrm{H}$, Major PW. Assessing the reliability of MRI-CBCT image registration to visualize temporomandibular joints. Dentomaxillofacial Radiology. 2015;44(6). doi: 10.1259/ dmfr.20140244

16. Al-Shwaikh H, Urtane I, Pirttiniemi P, Pesonen P, Krisjane Z, Jankovska I et al. Radiologic features of temporomandibular joint osseous structures in children with juvenile idiopathic arthritis. Cone beam computed tomography study. Stomatologija. 2016;18(2):51-60.

17. Kumar R, Pallagatti S, Sheikh S, Mittal A, Gupta D, Gupta S. Correlation Between Clinical Findings of Temporomandibular Disorders and MRI Characteristics of Disc Displacement. Open Dent J. 2015;9:273-81. doi: $10.2174 / 187421060150901027$

18. Keller H, Müller LM, Markic G, Schraner T, Kellenberger CJ, Saurenmann RK. Is early TMJ involvement in children with juvenile idiopathic arthritis clinically detectable? Clinical examination of the TMJ in comparison with contrast enhanced MRI in patients with juvenile idiopathic arthritis. Pediatr Rheumatol Online J. 2015;13:56. doi: 10.1186/s12969-015$\underline{0056-2}$

19. Junior CFM, Saito OC, Filho HAG. Avaliação ultrassonográfica dos distúrbios intracapsulares temporomandibulares. Radiol Bras. 2011 ;44(6):355-9. doi: 10.1590/S0100-39842011000600005

20. Al-Saleh MAQ, Alsufyani NA, Saltaji H, Jaremko JL, Major PW. MRI and CBCT image registration of temporomandibular joint: a systematic review. J Otolaryngol Head Neck Surg. 2016;45:30. doi: 10.1186/s40463-016-0144-4

21. Larheim TA, Abrahamsson AK, Kristensen M, Arvidsson LZ. Temporomandibular joint diagnostics using CBCT. Dentomaxillofac Radiol. 2015;44(1). doi: 10.1259/ dmfr.20140235

22. Watanabe PCA, Arita ES. Imaginologia e radiologia odontológica. Rio de Janeiro: Elsevier; 2012. 